\title{
Implante transaxilar del dispositivo de asistencia circulatoria Impella CP como puente al trasplante cardiaco: primera experiencia en España
}

Transaxillary implantation of the Impella CP mechanical circulatory support device as a bridge to heart transplant: first experience in Spain

David Couto-Mallón $^{\mathrm{a}, \mathrm{b}, \mathrm{c}}$, Francisco Estévez-Cid ${ }^{\mathrm{b}, \mathrm{d}}$, Miguel Solla-Buceta ${ }^{\mathrm{b}, \mathrm{e}}$, Carlos García-Velasco $^{\mathrm{b}, \mathrm{d}}$, María G. Crespo-Leiro, ${ }^{\mathrm{a}, \mathrm{b}, \mathrm{c}}$,José Joaquín Cuenca-Castillo, ${ }^{\mathrm{b}, \mathrm{c}, \mathrm{d}}$

\footnotetext{
${ }^{a}$ Servicio de Cardiología, Hospital Universitario de A Coruña, A Coruña, España

${ }^{b}$ Instituto de Investigación Biomédica de A Coruña (INIBIC), Universidad de A Coruña, A Coruña, España

${ }^{c}$ Centro de Investigación Biomédica en Red Cardiovascular (CIBERCV), Instituto de Salud Carlos III,

Madrid, España

${ }^{d}$ Servicio de Cirugía Cardiaca, Hospital Universitario de A Coruña, A Coruña, España

${ }^{e}$ Servicio de Medicina Intensiva, Hospital Universitario de A Coruña, A Coruña, España
} 


\section{Sr. Editor:}

El número de pacientes que llegan al trasplante cardiaco (TxC) en situación urgente y con dispositivos de asistencia circulatoria ha ido incrementándose con los años ${ }^{1}$.

El dispositivo de asistencia ventricular izquierda de corta duración Impella CP (Abiomed; Danvers, Massachusetts, Estados Unidos) es una bomba axial de flujo continuo que, atravesando la válvula aórtica, impulsa la sangre directamente del ventrículo izquierdo hacia la aorta ascendente ${ }^{2}$. El implante convencional es por acceso femoral a través de un catéter de 14 Fr y proporciona un flujo máximo teórico de $4 \mathrm{l} / \mathrm{min}$, aunque in vivo no se suele superar los 3,5 1/min. En la ficha técnica el tiempo de asistencia recomendado es $<7$ días. Este dispositivo ya se ha empleado con éxito en nuestro país como puente al $\mathrm{TxC}^{3}$. Según el registro de la Organización Nacional de Trasplantes, el $73 \%$ de los pacientes en urgencia 0 de TxC esperan al trasplante 10 días o menos ${ }^{4}$; sin embargo, el tiempo en espera de TxC de cada paciente es impredecible y ha ido en aumento los últimos años ${ }^{1}$. El acceso femoral obliga a inmovilizar al paciente durante la espera previa al TxC, lo que dificulta la rehabilitación y aumenta el riesgo de complicaciones tras el TxC.

Abiomed ha diseñado un kit para el implante transaxilar de los dispositivos Impella 2.5, Impella CP e Impella 5.0. Los estudios en humanos avalan el empleo del Impella 5.0 y el Impella $\mathrm{CP}$ para el tratamiento del shock cardiogénico ${ }^{3}$, si bien el Impella 5.0 requiere catéteres de mayor calibre (21 Fr), con un potencial riesgo de complicaciones vasculares.

Se presentan 4 casos de pacientes que recibieron un Impella CP como puente al TxC mediante abordaje transaxilar entre marzo y diciembre de 2016, a los que se siguió hasta el 31 de mayo de 2017. Se analizaron la supervivencia, el tiempo de ventilación mecánica después del TxC, los días de estancia en UCI y los eventos adversos como hemorragia o infección. El implante del Impella CP se realizó en quirófano a través de una incisión infraclavicular derecha para exposición de la arteria axilar. Después se realizaba la anastomosis terminolateral de un injerto de dacrón (10 mm) a la arteria, previa heparinización del paciente. A través del injerto, se introdujo el introductor del dispositivo, que se aseguró con el graft lock, y se progresó la guía rígida hasta el ventrículo izquierdo. El dispositivo se montó sobre la guía (sistema monorraíl) y se avanzó hasta su correcto posicionamiento, con control por fluoroscopia y ecocardiografía transesofágica (figura A).

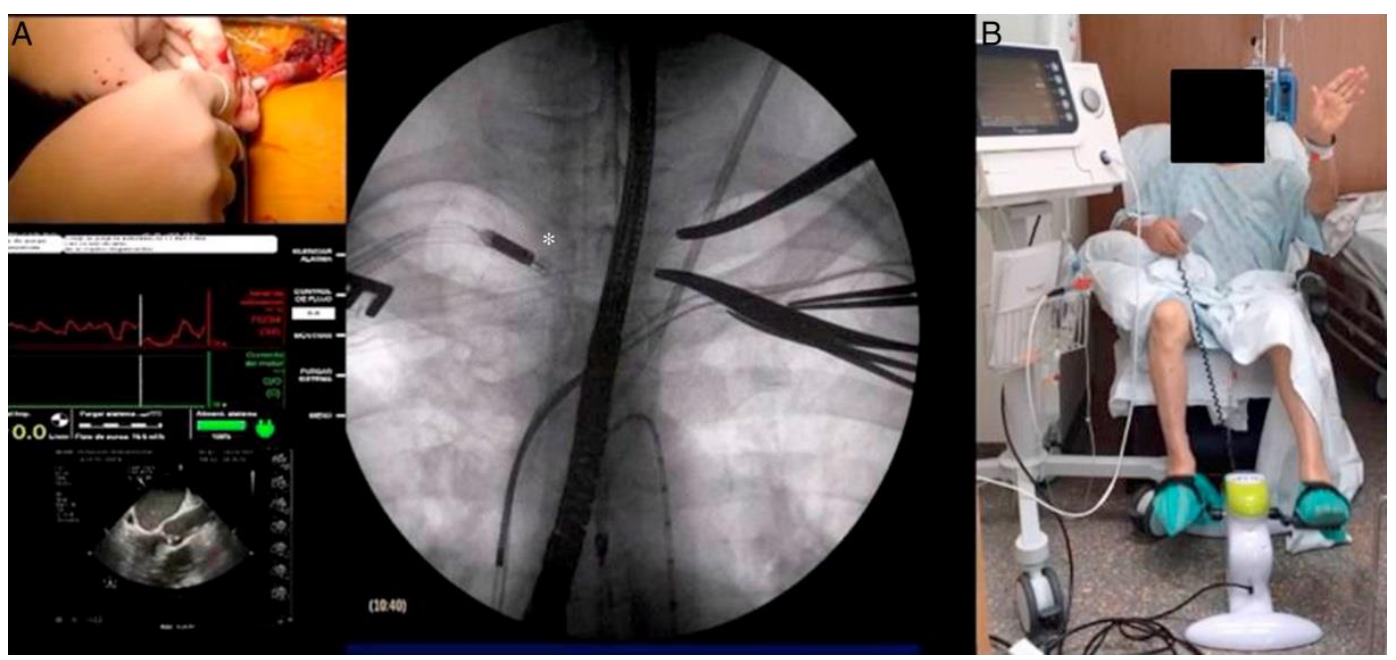

Figura. A: implante del dispositivo Impella CP guiado simultáneamente por fluoroscopia y por ecocardiografía transesofágica; se observa el avance de la bomba a nivel subclavio (asterisco). B: paciente realizando rehabilitación activa durante la asistencia con el dispositivo. 
Las características de los pacientes y las variables del estudio se muestran en la tabla. Se extubó a todos los pacientes después del implante del Impella CP y comenzaron el programa de rehabilitación precoz (cinesiterapia, fisioterapia respiratoria y movimientos activos) durante la asistencia con el dispositivo. La mediana de tiempo de asistencia fue de 13,5 [intervalo intercuartílico, 11,25-15,5] días. La anticoagulación se realizó con una solución de dextrosa al 5\% y heparina en concentración de $50 \mathrm{UI} / \mathrm{ml}$. El TxC se realizó con éxito, se dio el alta a los 4 pacientes con una media de estancia en UCI tras el TxC de 7,5 [5,5-9,75] días y una mediana al alta hospitalaria tras el TxC de 29,5 [26-33,5] días. Las principales complicaciones tras el TxC fueron 1 caso de taponamiento cardiaco a los 9 días tras el TxC y 1 ictus isquémico (1 paciente con estenosis carotídea bilateral significativa, que se recuperó sin secuelas). Tras una mediana de seguimiento de 363 días, todos los pacientes estaban vivos y en clase funcional I.

Tabla. Pacientes asistidos con Impella CP como puente al trasplante cardiaco

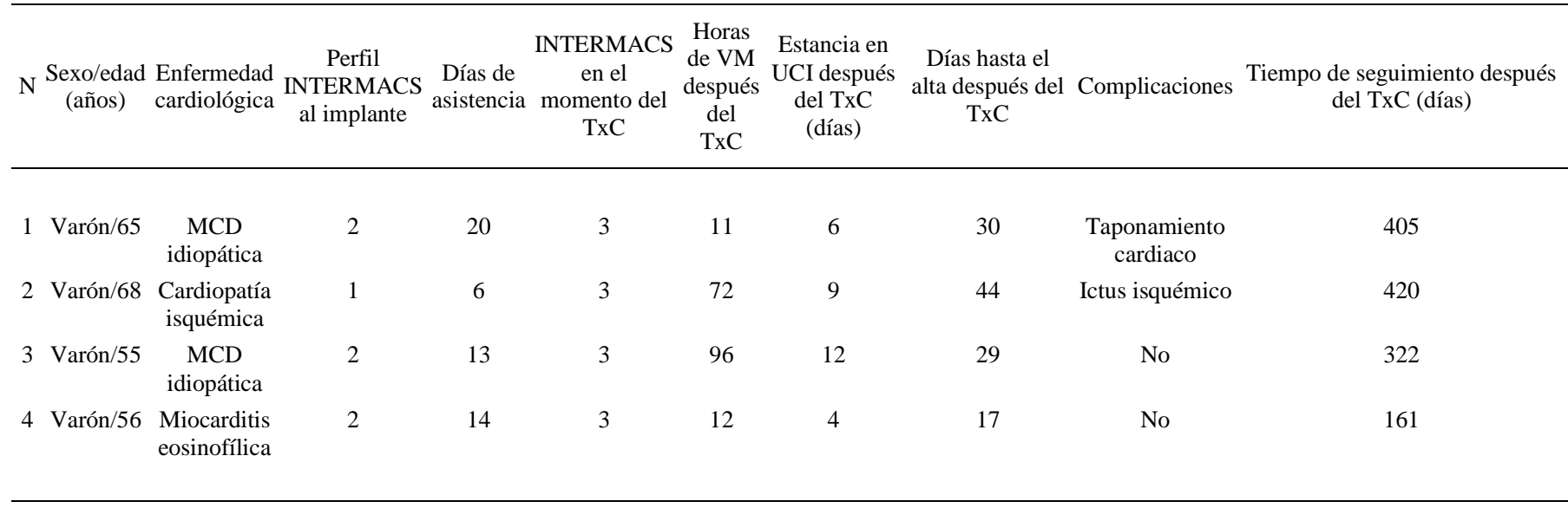

MCD: miocardiopatía dilatada; TxC: trasplante cardiaco; UCI: unidad de cuidados intensivos; VM: ventilación mecánica.

Hasta donde sabemos, se trata de la primera experiencia presentada en España del implante del Impella CP con acceso transaxilar como puente al TxC. Aunque la media de tiempo de asistencia fue superior al recomendado, no se observó disfunción del dispositivo y solo 1 paciente (número 3) presentó al décimo día de asistencia una hemolisis sin repercusión clínica, evidenciada por consumo de haptoglobina, que se resolvió ajustando las revoluciones del Impella CP. No hubo que recolocar ningún dispositivo por desplazamiento, probablemente debido al menor trayecto intravascular del Impella, que aumenta su estabilidad. Por lo tanto, se considera que el Impella CP es un dispositivo de asistencia ventricular izquierda de corto plazo muy útil para pacientes en urgencia 0 de $\mathrm{TxC}$ que presentan disfunción izquierda predominantemente.

El abordaje transaxilar realizado por equipos experimentados resulta seguro y permite la movilización y la fisioterapia precoz de estos pacientes (figura B), a diferencia del Impella CP transfemoral o el oxigenador extracorpóreo de membrana periférico, que obligan al paciente a permanecer en cama, con lo que se favorece la pérdida de masa muscular, se enlentece la recuperación funcional y aumenta el riesgo de complicaciones.

En nuestra serie, aunque pequeña, no se ha observado ninguna complicación relacionada directamente con el acceso transaxilar. El taponamiento cardiaco sucedió días después del TxC y se trató mediante pericardiocentesis. El paciente que sufrió el ictus isquémico no mostró focalidad neurológica durante la asistencia con el Impella $\mathrm{CP}$, y se interpretó como complicación relacionada con la cirugía de $\mathrm{TxC}$ en el contexto de enfermedad carotídea significativa. Estudios con mayor número de pacientes ayudarán a establecer con más claridad la utilidad de esta estrategia. 


\section{BIBLIOGRAFÍA}

1. González-Vílchez F, Segovia-Cubero J, Almenar L, et al. Registro Español de Trasplante Cardiaco. XXVII Informe Oficial de la Seccion de Insuficiencia Cardiaca y Trasplante Cardiaco de la Sociedad Española de Cardiologia (1984-2015). Rev Esp Cardiol. 2016;69:1071-1082.

2. Amat-Santos IJ, Varela-Falcón LH, Abraham WT. Terapias percutáneas en el tratamiento de la insuficiencia cardiaca aguda y crónica: presente y futuro. Rev Esp Cardiol. 2017;70:382-390.

3. Sayago I, Domínguez-Rodríguez F, Oteo-Dominguez JF, Gómez-Bueno M, Segovia J, AlonsoPulpón L. Dispositivo de asistencia circulatoria Impella CP W como terapia puente a trasplante cardiaco: primera experiencia en España. Rev Esp Cardiol. 2015;68:906-908.

4. ONT. Memoria de donación y trasplante 2015. Trasplante cardíaco [citado 13 Sep 2017]. Disponible en: http://www.ont.es/infesp/Memorias/ Memoria\%20corazo' n\%202015.pdf 